\title{
Spontaneous Retroperitoneal Hematoma in COVID-19 Severe Pneumonia-Dual-phase Multidetector Computed Tomography Angiogram and Role of Radiologist
}

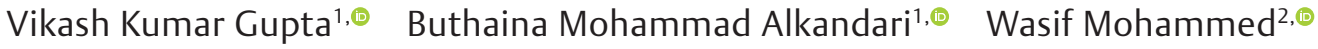 \\ Mohsen Ahmed Abdelmohsen ${ }^{1, \oplus}$ Mohammad Gaber Abdullah Mohammad ${ }^{1, \oplus}$ \\ 1Jaber Al Ahmad Hospital, Kuwait \\ Address for correspondence Vikash Kumar Gupta, \\ 2Sabah Hospital, Kuwait \\ MD (Radiodiagnosis), ATS Indirapuram, Ghaziabad 201014, India \\ (e-mail: vikash_k_gupta@yahoo.com).
}

J Clin Interv Radiol ISVIR 2021;6:58-60.

\begin{abstract}
Studies available in the literature have shown alterations in blood coagulation tests in severe cases of COVID-19 pneumonia, with a significant risk of venous thromboembo-

Keywords

- spontaneous retroperitoneal hematoma

- low-molecular weight heparin

- COVID-19 lism (VTE). Since microvascular thrombosis is a well-known fact in COVID-19 disease, requiring therapeutic anticoagulation, low-molecular weight heparin (LMWH) in prophylactic dose is a part of the clinical management of hospitalized COVID-19 patients. In this scenario, we describe three cases of abdominal spontaneous retroperitoneal hematoma $(\mathrm{SRH})$ in hospitalized reverse transcriptase-polymerase chain reaction (RT-PCR)-confirmed COVID-19 patients.
\end{abstract}

\section{Introduction}

Severe acute respiratory syndrome Coronavirus 2 (SARS-CoV-2) was initially reported to affect the respiratory system, with severe cases causing acute respiratory distress syndrome (ARDS), leading to multiorgan failure and death. As the pandemic grew, multiple reports emerged where patients with moderate-to-severe COVID-19 presented with alterations in blood coagulation profile and increased risk of thromboembolic phenomenon. ${ }^{1}$ Low-molecular weight heparin (LMWH) was used prophylactically and therapeutically for the management of such patients. One of the recognized complications of LMWH is spontaneous extraperitoneal hemorrhage (SEH) or spontaneous retroperitoneal hemorrhage (SRH). We describe the clinical details and management strategy of three COVID-19 patients below, presenting as SEH or SRH.

published online June 14, 2021
DOI https://doi.org/

$10.1055 / \mathrm{s}-0041-1728970$

ISSN 2457-0214

\section{Case 1}

A 63-year-old Indian gentleman with severe COVID-19 pulmonary manifestation was on invasive mechanical ventilation in ICU for hypoxia (arterial PO2 74\%). Anticoagulant (subcutaneous LMWH $0.5 \mathrm{mg} / \mathrm{kg}$ body weight per day) in prophylactic dose was administered in addition to other drugs as per the Ministry of Health protocol for serious COVID-19 patients. The patient developed abdominal distention 1 week after his admission. His hemoglobin level dropped from 14.1 to $7.2 \mathrm{mg} / \mathrm{dL}$.

A contrast-enhanced computed tomography (CECT) revealed a large abdominopelvic hematoma on the left side with active contrast extravasation ( - Fig. 1). It was decided to treat endovascularly. On angiography through right femoral access, an active contrast extravasation was seen from a branch of the anterior division of the left internal iliac artery,

(c) 2021. Indian Society of Vascular and Interventional Radiology. This is an open access article published by Thieme under the terms of the Creative Commons Attribution-NonDerivative-NonCommercial-License, permitting copying and reproduction so long as the original work is given appropriate credit. Contents may not be used for commercial purposes, or adapted, remixed, transformed or built upon. (https://creativecommons.org/licenses/by-nc-nd/4.0/).

Thieme Medical and Scientific Publishers Pvt. Ltd. A-12, 2nd Floor, Sector 2, Noida-201301 UP, India 

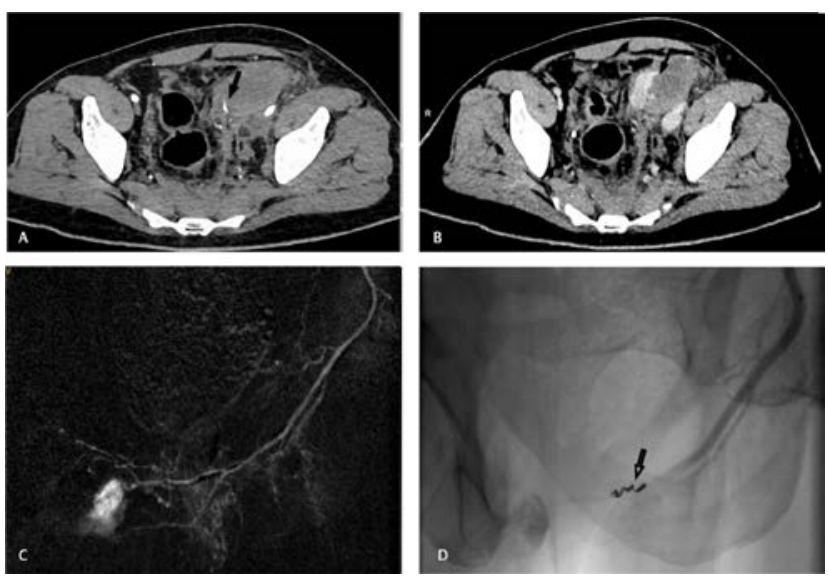

Fig. 1 A large left abdominopelvic extraperitoneal hematoma. Contrast-enhanced computed tomography (CECT) axial, arterial phase image (A) showing the hematoma as an ill-defined hypodensity with focal contrast extravasation (black arrow). Three minutes delayed phase axial CT image (B) showing pooling of contrast (arrow). Digital subtraction angiography (DSA) (c) showing contrast extravasation from a branch of the left internal iliac artery (obturator artery). Postembolization angiogram (D) demonstrating coil embolization of the arterial branch with cessation of the extravasation.
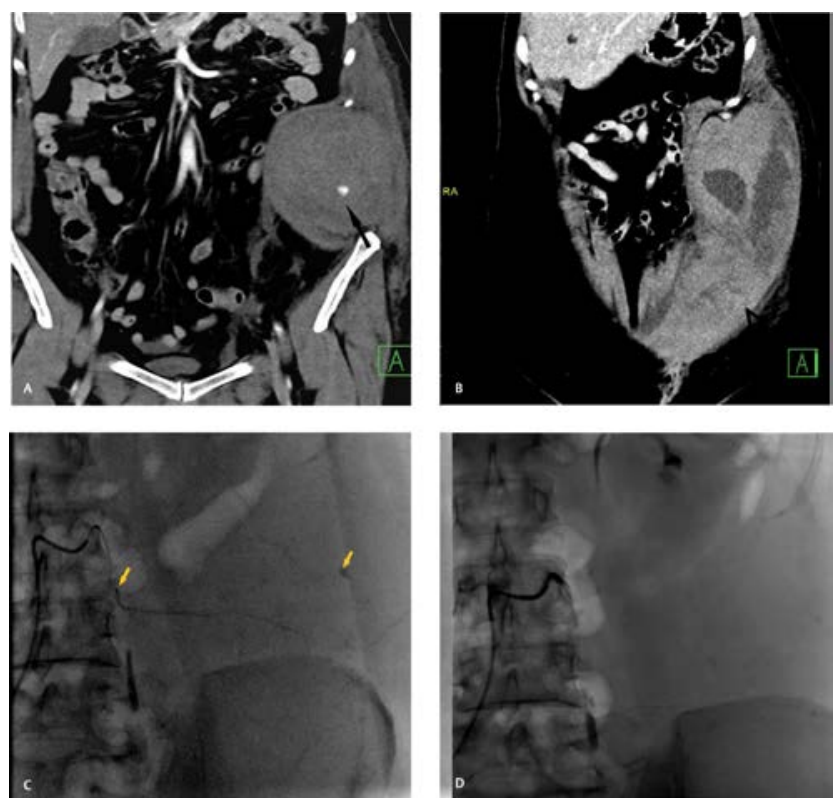

Fig. 2 A large abdominal wall hematoma on the left side. Contrast-enhanced computed tomography (CECT) coronal image in the arterial phase (A) and 3 minutes delayed phase (B) showing the hematoma as a large hypodense lesion involving the abdominal wall muscles with contrast extravasation (arrow in $\mathbf{A}$ ) and pooling (arrow in B) (arrow). Angiogram of left 3rd lumbar artery (C) demonstrating active extravasation of contrast (arrow). Postembolization angiogram (D) showing occlusion of the branch.

assumed to be obturator artery. Selective cannulation was done with a $2.8 \mathrm{~F}$ Progreat microcatheter (Terumo; Somerset, New Jersey, USA). Embolization of the artery was performed with 2 to $4 \mathrm{~mm}$ diameter 0.018" coils (Cook Medical, Bloomington, Indiana, United States) successfully ( - Fig. 1). The hemoglobin values were stable during immediate postprocedure period. However, he succumbed due to cardiac arrest 3 days later.

\section{Case 2}

A 57-year-old Kuwaiti lady presented with complaints of fever, cough, and vomiting. Screening for COVID-19 was positive. The treatment protocol with other medications included a prophylactic dose of anticoagulant (subcutaneous LMWH $0.5 \mathrm{mg} / \mathrm{kg}$ body weight per day). On the sixth day after admission, she complained of severe abdominal pain with a sudden drop of serum hemoglobin level from 12 to $9 \mathrm{mg} / \mathrm{dL}$.

A CECT revealed a large extraperitoneal abdominopelvic hematoma on the left side (-Fig. 2). A diagnostic angiography revealed contrast extravasation from the left 3rd lumbar artery. Superselective catheterization with embolization of the bleeding artery was done with gel foam (-Fig. 2). She had an uneventful recovery.

\section{Case 3}

A 63-year-old Kuwaiti gentleman presented with complaints of shortness of breath and cough with stable vital parameters and arterial PO2 95\% on room air. He was diagnosed to have COVID-19.

LMWH $0.5 \mathrm{mg} / \mathrm{kg}$ body weight per day was administered subcutaneously. He complained of severe abdominal pain on the third day of his admission. He had abdominal distention, tachypnea with a drop in systolic blood pressure to $70 \mathrm{mmHg}$. The hemoglobin level dropped from 15 to $8.9 \mathrm{~g} / \mathrm{dL}$.

CECT revealed thickened edematous left psoas muscle and adjoining large extraperitoneal hematoma extending to the left anterolateral abdominal wall ( - Fig. 3).
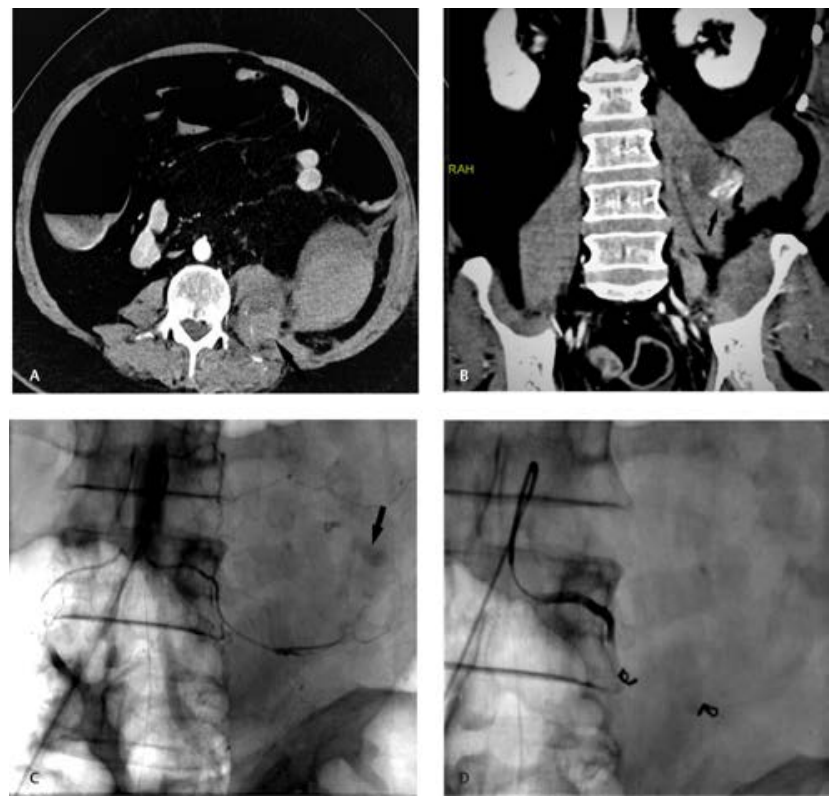

Fig. 3 A large left retroperitoneal hematoma. Contrast-enhanced computed tomography (CECT) axial image in the arterial phase (A) showing a large hematoma in the left psoas, quadratus lumborum, and adjacent retroperitoneum with contrast extravasation. Three minutes delayed phase CECT coronal image (B), showing further pooling of contrast (arrow). Angiogram of the left 4th lumbar artery pre-embolization (C) showing contrast extravasation (arrow in $\mathbf{C}$ ) and postembolization angiogram (D) showing the coils and successful occlusion of the artery. 
Contrast extravasation was seen from the left 4th lumbar artery, which was successfully embolized with coils, (-Fig. 3 ) similar to case 1 . He gradually improved and was discharged from the hospital after a week.

\section{Discussion}

Spontaneous intramuscular bleeding in COVID-19 patients is likely to be multifactorial. Such hematomas are a known complication of LMWH therapy. ${ }^{2}$ Cough, a common symptom of COVID-19 pneumonia, may increase intra-abdominal pressure, resulting in arterial rupture. ${ }^{3}$ Invasive ventilation with continuous positive pressure ventilation (CPAP) is also likely to increase intra-abdominal pressure. ${ }^{4}$ Retroperitoneal hemorrhage has also been described as a rare and severe complication in patients undergoing extracorporeal membrane oxygenation. ${ }^{5}$ Early suspicion by clinical evaluation, confirmation by CECT scan, and endovascular embolization may be life-saving in these scenarios.

\section{Conclusion}

Severe COVID-19 pneumonia patients are treated with prophylactic or higher doses of LMWH. SEH may be suspected in patients with sudden localized pain and drop in hematocrit. CECT is the imaging of choice to localize the site of bleeding and plan the endovascular treatment. Embolization in such patients is safe and effective.

\section{Conflict of Interest}

None declared.

\section{References:}

1 Xiong M, Liang X, Wei Y-D. Changes in blood coagulation in patients with severe coronavirus disease 2019 (COVID-19): a meta-analysis. Br J Haematol 2020;1 89(6):1050-1052

2 Chung KT. Intra-abdominal hematoma following enoxaparin injection. Clin Med Insights Case Rep 2016;9:35-38

3 Jang JY, Lim YS, Woo JH, Jang JH. Spontaneous rupture of intercostal artery after severe cough. Am J Emerg Med 2015;33(1): 131.e1-131.e3

4 Yam LY, Chen RC, Zhong NS. SARS: ventilatory and intensive care. Respirology 2003;8(Suppl):S31-S35

5 Zhang J, He X, Hu J, Li T. Retroperitoneal hemorrhage during veno-venous extracorporeal membrane oxygenation in COVID-19 Patients: clinical experience and review of literature. Research Square 2020. Doi: 10.21203/rs.3.rs-32685/v1 